\section{P166 PREVALENCE OF CHLAMYDIA AMONG PREGNANT WOMEN, GYNECOLOGY CLINIC ATTENDEES, AND SUBFERTILE WOMEN IN GUANGDONG, CHINA:A CROSS SECTIONAL SURVEY}

C Li*, W Tang, C Wang, B Yang. Dermatology Hospital of Southern Medical University, Guangzhou, China

10.1136/sextrans-2021-sti.267

Background Chlamydia is a major cause of infertility, but its epidemiology among women of reproductive age remains unclear in China. This study investigated the prevalence of chlamydia and associated factors among Chinese women aged 18-44 years who were either 1) pregnant; 2) attending gynaecology clinics; or 3) subfertile.

Methods We conducted a cross-sectional survey and recruited participants from obstetrics, gynaecology, and infertility clinics in Guangdong, between March to December, 2019. We collected information on individuals' socio-demographic characteristics, previous medical conditions, and sexual behaviours. First-pass urine and cervical swabs were tested using nucleic acid amplification testing. We calculated the prevalence in each population and subgroup by age, education, and age at first sex. Multivariable binomial regression models were used to identify factors associated with chlamydia prevalence.

Results We recruited 1730 participants, including 881 pregnant women, 595 gynaecology clinic attendees, and 254 subfertile women. The overall prevalence was 6.7\% (95\% Confidence Interval (CI): 5.2\%-8.5\%), 8.2\% (95\%CI:6.2\%-10.7\%), 5.9\% (95\%CI: $3.5 \%-9.3 \%$ ) for the above three populations, respectively. The subgroup-specific prevalence was highest among those who first had sex before 25 years and older pregnant women (>35 years). The proportion of asymptomatic chlamydia was $85 \%, 40 \%$, and $60 \%$ among pregnant women, gynaecology clinic attendees, and subfertile women, respectively. Age at first sex ( $<25$ years), multipara, and ever having more than one partner increased the risk of chlamydia.

Conclusion Women of reproductive age in China have a high prevalence of chlamydia. As most women with chlamydia were asymptomatic, routine chlamydia screening is urgently needed in China.

\section{P167 IMPACT OF COVID-19 ON ADOLESCENT GIRLS AND YOUNG WOMEN IN A COMMUNITY-BASED HIV PREP PROGRAMME IN SOUTH AFRICA}

\begin{abstract}
1,2,3R Peters ${ }^{*},{ }^{1,4} \mathrm{~A}$ Medina-Marino, ${ }^{5} \mathrm{C}$ Celum, ${ }^{4} \mathrm{~L}$ Bekker. ${ }^{1}$ Foundation For Professional Development, East London, South Africa; ${ }^{2}$ University of Pretoria, Department of Medical Microbiology, Pretoria, South Africa; ${ }^{3}$ University of Cape Town, Division of Medical Microbiology, Cape Town, South Africa; ${ }^{4}$ Desmond Tutu Health Foundation, Cape Town, South Africa; ${ }^{5}$ University of Washington, Department of Global Health, Seattle, USA
\end{abstract}

10.1136/sextrans-2021-sti.268

Background The coronavirus (COVID-19) pandemic has severely impacted healthcare service delivery, including preexposure prophylaxis (PrEP) worldwide. COVID-19 may increase the vulnerability of adolescent girls and young women (AGYW). We investigated the impact of COVID-19 on HIV, STIs and pregnancy in a community-based PrEP programme for AGYW in South Africa.
Methods We used data from an ongoing study of strategies to improve the prevention-effective use of PrEP among AGYW living in a peri-urban and a rural community in the Eastern Cape, South Africa. PrEP services have been provided since October 2018; AGYW are expected to attend monthly PrEP service activities with Xpert $^{\circledR}$ testing for STIs at the 6-, 12and 24-months visits. PrEP services were partially impacted during the South African lockdown (March-April 2020). An observational analysis of PrEP service utilization and HIV, STI and pregnancy results was conducted comparing data from before COVID-19 introduction and during the COVID-19 epidemic in South Africa.

Results A total of 546 AGYW attending PrEP services for $\geq 3$ months were included in our analysis. The number of AGYW visits for PrEP and HIV testing dropped by $29 \% \quad(\mathrm{p}<0.001)$ during the COVID-19 epidemic in South Africa. HIV test positivity increased from $0.54 \%$ to $1.94 \%$ during the COVID-19 epidemic $(p=0.03)$. STI test positivity increased from 23 to $30 \% \quad(p=0.20)$ for Chlamydia trachomatis, $7 \%$ to $14 \%$ for Neisseria gonorrhoeae $(p=0.08)$, and 8 to $12 \%$ for Trichomonas vaginalis $(p=0.32)$. Pregnancy test positivity increased from $1.2 \%$ before to $4.1 \%$ during the COVID-19 epidemic $(\mathrm{p}=0.002)$.

Conclusion South African AGYW substantially decreased their visits for PrEP in a community-based PrEP study during the COVID-19 epidemic, while rates of new HIV infection, STIs, and pregnancy increased, indicating unprotected sex. The impact of COVID-19 on healthcare services, the economy and social interactions has enhanced the vulnerability and health risks of AGYW in our setting.

\section{P171 FROM TRIAL TO PROGRAM: TTANG02 SCALE-UP AND IMPLEMENTATION SUSTAINS STI POINT-OF-CARE TESTING IN REGIONAL AND REMOTE AUSTRALIAN ABORIGINAL HEALTH SERVICES}

${ }^{1} \mathrm{~L}$ Causer*, 'L Watchirs-Smith, ${ }^{1} \mathrm{~A}$ Saha, ${ }^{1} \mathrm{H}$ Wand, ${ }^{1} \mathrm{~K}$ Smith, ${ }^{1} \mathrm{~S}$ Badman, ${ }^{1} \mathrm{~B}$ Hengel, ${ }^{2} \mathrm{~K}$ Andrewartha, ${ }^{2} \mathrm{~J}$ Richards, ${ }^{1,3} \mathrm{~A}$ Tangey, ${ }^{4} \mathrm{~K}$ Hawkett, ${ }^{5} \mathrm{C}$ Carroll, ${ }^{6} \mathrm{~S} \mathrm{O}^{\prime} \mathrm{Connor}$, ${ }^{7} \mathrm{R}$ Marshal-Lang, ${ }^{8} \mathrm{E}$ Moore, ${ }^{2} \mathrm{M}$ Shephard, ${ }^{1} \mathrm{R}$ Guy, on behalf of the TTANGO2 Collaboration. 'The Kirby Institute, UNSW Sydney, Sydney, Australia; ${ }^{2}$ Flinders University International Centre for Point-of-Care Testing, Bedford Park, Australia; ${ }^{3}$ Ngaanyatjarra Health Service, Australia; ${ }^{4}$ Aboriginal Health Council of Western Australia, Perth, Australia; ${ }^{5}$ Aboriginal Health Council of South Australia, Adelaide, Australia; ${ }^{6}$ Queensland Health, Townsville, Australia; ${ }^{7}$ Communicable Disease Control Directorate, Department of Health, Perth, Australia; ${ }^{8}$ Aboriginal Medical Services Alliance Northern Territory, Alice Springs, Australia

\subsection{6/sextrans-2021-sti.269}

Background Young people living in remote Aboriginal communities experience some of the highest rates of chlamydia (CT) and gonorrhoea (NG) infection globally. A cluster-randomised controlled trial (TTANGO) in 11 remote primary health services demonstrated point-of-care (POC) testing for CT/NG was acceptable, accurate, improved the uptake and timeliness of treatment, and was cost-saving. Subsequently, POC testing was scaled-up and implemented in a further 20 remote health services (TTANGO2 program) across four jurisdictions (31 in total). We determine whether the uptake of POC testing observed in the trial was also achieved and sustained during the long-term program.

Methods We conducted descriptive, interrupted time series and trajectory analyses to compare POC testing patterns over two 